Manuelle Medizin 2017 ·55:171

DOI 10.1007/s00337-017-0262-8

Online publiziert: 2. Mai 2017

๑) Springer Medizin Verlag GmbH 2017

CrossMark

\author{
A. Wienke \\ Wienke \& Becker - Köln, Köln, Deutschland
}

\section{Chirotherapeutische Behandlung durch Masseure unzulässig}

Masseure bzw. medizinische Bademeister dürfen manuelle Therapie nicht durchführen und nicht als eigene Leistungen abrechnen. Dies hat das Bundessozialgericht (BSG) gleich in mehreren Parallelverfahren klargestellt. Die auf Landesebene dazu abgeschlossenen Rahmenvereinbarungen seien nicht zu beanstanden, wenn diese ausschließlich den Physiotherapeuten, nicht aber den Masseuren bzw. medizinischen Bademeistern die Durchführung und Abrechnung manueller Therapie erlaube. Nur aufgrund von Bestandsschutzregelungen dürften Masseure und medizinische Bademeister heute noch Leistungen der manuellen Therapie abrechnen.

Die Entscheidung des BSG fiel eindeutig aus: Die Berechtigung zur Leistung der manuellen Therapie und deren Abrechnungserlaubnis werde nur an Physiotherapeuten erteilt, die eine spezielle Weiterbildung in manueller Therapie abgeschlossen hätten. Masseure und medizinische Bademeister seien dagegen auch mit absolvierter Weiterbildung in manueller Therapie dazu nicht befugt. Das BSG beruft sich dabei auf die Regelungen in der Heilmittel-Richtlinie ( $\$ 17$ Abs. 2 und $\$ 19$ Abs. 3 Nr. 7) des Gemeinsamen Bundesausschusses (B-GA), nach denen die manuelle Therapie Teil der Bewegungstherapie und nur im Weiterbildungsprogramm des Physiotherapeuten vorgesehen ist. Hingegen ist sie nicht als Fortbildungsmöglichkeit im Bereich Massagetherapie gelistet.

Mit dieser Zuordnung des Verordnungsgebers werde nach Auffassung des BSG auch nicht in den vom Grundgesetz geschützten Kernbereich der Tätigkeit der Masseure und medizinischen Bademeister eingegriffen. Auch wenn diese von der Leistungserbringung im Bereich der manuellen Therapie ausgeschlossen würden, handele es sich dabei lediglich um eine Berufsausübungsregelung, die durch schützenswerte Interessen des Gemeinwohls gerechtfertigt sei. Ferner stellt das BSG darauf ab, dass der Ausbildungsrückstand der Masseure gegenüber den Physiotherapeuten im Hinblick auf Bewegungstherapie durch eine Weiterbildung nicht kompensiert werden könne. Der Gesetzgeber habe Inhalt und Grenzen der hier betroffenen Ausbildungsberufe im Einklang mit der Berufsfreiheit (Art. 12 GG) festgelegt und dabei die Trennung der Bereiche Massage/medizinisches Badewesen und Physiotherapie beibehalten, sodass zwei voneinander völlig getrennte Ausbildungsberufe entstanden seien, deren Ausbildungsziele, -inhalte und -dauer sich voneinander unterscheiden.

Die Berufswahlfreiheit der Masseure und medizinischen Bademeister sei nicht betroffen, weil nicht der Zugang zum Beruf eingeschränkt werde, sondern lediglich Inhalt, Umfang und Modalitäten des Berufs des Masseurs bzw. medizinischen Bademeisters tangiert würden. Es gehe nur um die Erbringung einer speziellen Leistung, der manuellen Therapie, nicht um den Zugang zum Beruf als solchen oder um wesentliche Kernleistungen, ohne die eine sinnvolle Ausübung des Berufs nicht möglich wäre. Der Gesetzgeber habe Inhalt und Grenzen der hier betroffenen Ausbildungsberufe ohne Verstoß gegen die Berufsausübungsfreiheiten festgelegt.
Die Entscheidung des BSG ist $\mathrm{zu}$ begrüßen. Sie ist Ausprägung der gesetzgeberischen Wertungen im Hinblick auf die Trennung zwischen Massage- und Physiotherapie. Vor dem Hintergrund der unterschiedlichen Ausbildungen und schwerpunktmäßigen Tätigkeiten ist es erfreulich, dass auch Patienten nun Rechtssicherheit haben und nach den vom G-BA geforderten fachlichen Kenntnissen und Fähigkeiten behandelt werden können.

\section{Korrespondenzadresse}

\section{Dr. A. Wienke}

Wienke \& Becker - Köln

Sachsenring 6, 50677 Köln, Deutschland

AWienke@Kanzlei-WBK.de

\section{Einhaltung ethischer Richtlinien}

Interessenkonflikt. A. Wienke gibt an, dass kein Interessenkonflikt besteht.

Dieser Beitrag beinhaltet keine vom Autor durchgeführten Studien an Menschen oder Tieren. 